\title{
An in vivo comparison of the efficacy of hemostatic powders, using two porcine bleeding models
}

This article was published in the following Dove Press journal: Medical Devices: Evidence and Research

\author{
Melinda $\mathrm{H}$ MacDonald \\ Allen Y Wang \\ Jeffrey W Clymer \\ Richard W Hutchinson \\ Richard Kocharian \\ Ethicon Inc., Somerville, NJ, USA
}

Correspondence: Jeffrey W Clymer Ethicon Inc., 4545 Creek Road,

Cincinnati, OH 45242, USA

$\mathrm{Tel}+\mathrm{I} 5133373318$

Email jclymer@its.jnj.com

\begin{abstract}
Background: Usage of topical hemostatic agents in surgery is increasing, including use during minimally invasive procedures, and even for surgeries that have a low risk of bleeding complications. A novel product, Surgice ${ }^{\circledR}$ Powder - Absorbable Hemostatic Powder (SP), made from oxidized regenerated cellulose (ORC) fabric, has been developed for adjunctive use in surgical procedures to assist in control of oozing bleeding over broad areas and where access could be difficult with a fabric ORC product. This study compares the new SP to other commercially available hemostatic powder products in two in vivo models.

Methods: Hemostatic efficacy of SP was compared to two polysaccharide-based hemostats in a porcine liver punch biopsy model and to three polysaccharide-based hemostats and one nonregenerated oxidized cellulose hemostat in a porcine liver abrasion model. Primary outcomes measured were hemostatic efficacy, defined as hemostasis within 10 minutes of application, and time-to-hemostasis (TTH).

Results: In the punch biopsy model, SP displayed significantly higher effective hemostasis rates than one of the polysaccharide hemostats $(p=0.047)$ and faster TTH than both $(p<0.001)$. In the liver abrasion model, SP had significantly higher effective hemostasis rates $(p \leq 0.002)$ and faster TTH $(p<0.001)$ than the other four hemostatic agents. The amount of powder applied within the ranges used did not appear to affect hemostatic efficacy.

Conclusion: In both the liver punch biopsy model of mild to moderate bleeding and the liver abrasion model of mild but diffuse oozing, SP provided more effective hemostasis and faster TTH than other marketed hemostatic powders. The results from this in vivo study suggest that Surgicel Powder may be useful in clinical applications where control of oozing capillary, mild venous, and small arterial hemorrhage is required including bleeding in difficult-to-access locations.
\end{abstract}

Keywords: hemostasis, oxidized regenerated cellulose, Surgicel, in vivo model of bleeding, hemostatic powder, absorbable hemostat, topical absorbable hemostat

\section{Introduction}

Intraoperative bleeding can manifest in many forms and present significant perioperative morbidity. ${ }^{1}$ The selection of appropriate methods and products for control of bleeding is dependent on diverse factors including bleeding intensity, anatomical location, visibility, and access to the source of the bleeding. For mild to moderate bleeding, products based on cellulose or other polysaccharides have been gaining popularity as adjunctive hemostats due to their ease of use and efficacy. For example, one of the most commonly used topical hemostatic agents is Surgicel ${ }^{\circledR}$ Original Absorbable Hemostat (SF), a fabric consisting of oxidized regenerated cellulose (ORC). ${ }^{2}$ 
The mechanism of action for accelerated clotting by ORC hemostats, including SF, is not completely understood, but there appears to be a physical effect with the ORC absorbing water, swelling slightly to provide tamponade, and aid in the formulation of a clot. ${ }^{3}$ The ORC fibers trap fluid, blood proteins, platelets, and cells forming a mechanical scaffold, which acts as a barrier to blood flow and as a matrix for solid fibrin clot formation. ${ }^{3}$

Recently, Surgicel ${ }^{\circledR}$ Powder - Absorbable Hemostatic Powder (SP), a new topical absorbable hemostat, has been developed for use in both open and laparoscopic surgery. The powder is produced from the same ORC fabric material that is used in SF. SP is specifically intended to be used for treatment of broad surface, mild bleeding or oozing, and adjunctively in surgical procedures to assist in the control of capillary, venous, and small arterial hemorrhage when ligation or other conventional methods of control are impractical or ineffective. The product is ready to use out of the package with no special preparation required.

Several other powdered hemostatic products are currently available in the US and European markets, including Arista ${ }^{\mathrm{TM}}$ AH Absorbable Hemostatic Particles (AR), PerClot ${ }^{\circledR}$ Polysaccharide Hemostatic System (PC), Traumastem P Sterile Resorbable Haemostatic Powder (TP), and Instantaneous ${ }^{\circledR}$ Compound Microporous Polysaccharide Haemostatic Powder (IP). AR is a plant-based hemostatic cellulose powder derived from purified plant starch and is currently the predominant powdered hemostatic product in the US. ${ }^{4} \mathrm{PC}$ is another plant starch derived powdered hemostatic product composed of polysaccharide particles and is currently available for use in Europe. ${ }^{5} \mathrm{TP}$ is composed of a hydrogen calcium salt of oxidized non-regenerated cellulose and is marketed in Europe. IP is a polysaccharide powder product available in China.

A few studies have evaluated the hemostatic efficacy of fabric forms of the Surgice ${ }^{\circledR}$ Family of Absorbable Hemostats relative to either AR or PC, and no published study has compared the new powder version of SF to other adjunctive hemostatic products. This is the first study designed to compare the performance of SP in standardized preclinical bleeding models to the performance of other powder hemostatic products. The objective of this study was to determine whether there are significant differences in hemostatic efficacy between these products when used in vivo in focused mild to moderate bleeding (swine liver punch biopsy) and diffuse mild bleeding (swine liver abrasion) models.

\section{Methods}

All in vivo procedures were reviewed, and animals were approved for use by the Ethicon Institutional Animal Care and Use Committee in compliance with the US Animal
Welfare Act Regulations (9CFR, Parts 1, 2, and 3) and the Guide for the Care and Use of Laboratory Animals of the Association for Assessment and Accreditation of Laboratory Animal Care, International. Animals used were female Yorkshire swine weighing $54.2-74.1 \mathrm{~kg}$ ( $\mathrm{n}=5$ per study) supplied by Animal Biotech Industries, Inc., Danboro, PA, USA. Procedures followed were similar to those previously reported, ${ }^{6}$ but modified to examine powder application by changing from a spleen to liver punch biopsy and abrasion models.

SP was obtained from Ethicon, Inc. (Somerville, NJ, USA). AR was obtained from Medafor ${ }^{\circledR}$ Hemostatic Polymer Technologies (Minneapolis, MN, USA). PC was obtained from CryoLife, Inc. (Kennesaw, GA, USA). TP was obtained from Bioster, A.S., (Veverská Bítýška, Czech Republic). IP was obtained from Shandong Success Pharmaceutical Technology Co, (Jinan, Shandong, People's Republic of China). The negative control treatment, used in both models to confirm the validity of the model, involved application of a TELFA ${ }^{\mathrm{TM}}$ "Ouchless" non-adherent pad, obtained from Covidien (Minneapolis, MN, USA). For the punch biopsy trials, powder samples were pre-weighed as 75 or $125 \mathrm{mg}$ dosages, and applied directly to the bleeding defects under a Telfa dressing. For the abrasion trials, $300 \mathrm{mg}$ masses of powder were used. These masses were determined prior to the study by selecting an appropriate mass of powder required to adequately cover the defect area. In addition, for the punch biopsy evaluations a second mass of powder was determined that would cover the defect with some excess available to distribute as required.

For both models, intramuscular butorphanol tartrate $(0.1-0.3 \mathrm{mg} / \mathrm{kg})$ was given pre-operatively and repeated approximately $3 \frac{1}{2}$ to 4 hours after the initial dose to provide appropriate analgesia. Each pig was anesthetized with an intramuscular injection of tiletamine $(5.0 \mathrm{mg} / \mathrm{kg})$, xylazine $(5.0 \mathrm{mg} / \mathrm{kg})$, and glycopyrrolate $(0.01 \mathrm{mg} / \mathrm{kg})$. Propofol injectable emulsion (1\%) was administered to effect when necessary to facilitate intubation. When an appropriate plane of anesthesia was achieved, an endotracheal tube was inserted, and inhalation anesthesia was maintained throughout the remainder of the procedure via semi-closed circuit inhalation of isoflurane (1-4\%) mixed with $100 \%$ oxygen at a flow rate of 1-2.5 liters/minute. Mechanical ventilation at 9-16 respirations/minute with a tidal volume of 10-20 $\mathrm{mL} / \mathrm{kg}$ was used to ensure adequate ventilation. Lactated Ringer's Solution was administered intravenously at a rate of $10-15 \mathrm{~mL} / \mathrm{kg} / \mathrm{hr}$ throughout the procedure. Heart rate, respiratory rate, peripheral capillary oxygen saturation $\left(\mathrm{SpO}_{2}\right)$, electrocardiography (ECG), $\mathrm{CO}_{2}$, body temperature, and 
color of mucous membranes were monitored during surgery to ensure an adequate anesthetic plane.

A ventral midline abdominal incision was performed and the cranial portion of the midline incision was extended subcostally bilaterally to create a chevron pattern incision using Harmonic ${ }^{\circledR}$ Focus+ Shears and Harmonic ${ }^{\circledR}$ Synergy Blades (Ethicon, Cincinnati, OH, USA) to improve exposure of the liver. The liver was positioned as necessary to maximize testing surface availability. The abdominal organs were kept moist with saline and saline-soaked laparotomy sponges throughout the procedure.

For the punch biopsy model, liver parenchymal defects were created on the diaphragmatic surface of accessible areas of the left, right, and quadrate lobes using a disposable $6 \mathrm{~mm}$ biopsy punch with a depth stop set to $3 \mathrm{~mm}$. Each animal provided 14 sites that were randomly used for two applications of each product at each level of applied mass plus two negative controls. The core portion of the biopsy was grasped and sharply dissected free from the underlying surface causing mild to moderate hemorrhage. For the abrasion model, a section of the diaphragmatic surface of accessible areas of the left, right and quadrate lobes, approximately $3 \mathrm{~cm} \times$ $3 \mathrm{~cm}$, was abraded by scraping with a cautery tip cleaner. Each animal provided 14 sites that were randomly used for three applications of each product plus two negative controls. The abrasion extended through the visceral peritoneum and fibrous liver capsule to expose the liver parenchyma and cause mild hemorrhage or oozing.

For both models, the defect site was allowed to bleed for several seconds prior to product application to allow for characterization of the resulting hemorrhage. Bleeding at each defect site was classified as either mild (capillary, arteriole, or venule oozing) or moderate (flowing venous and/or arterial bleeding) at the time of wound creation and prior to treatment. No defects were classified as having severe bleeding (pulsatile or spurting arterial or high volume venous bleeding) during this study. The study surgeon was blinded to treatment identity until after the bleeding classification was recorded. The trial site was blotted with gauze and then one of the test articles or the negative control treatment was applied.

For the purposes of this study, effective hemostasis was defined as the cessation of free flow bleeding within 10 minutes following treatment and maintenance of hemostasis without occlusive pressure over a subsequent 1-minute evaluation period. Pinpoint or petechial bleeding that appeared but did not grow was not considered to be free flow bleeding. Time-to-hemostasis (TTH) was defined as the time at which the last tamponade was discontinued for those sites scored as effectively hemostatic. Defect trial sites aborted as failures were recorded as having a TTH of $>10$ minutes. One negative control was applied at the beginning and end of testing for each animal. Confirmation that tamponade alone did not control bleeding from these two sites within a 10-minute period demonstrated consistency of the experimental model over the testing period. The order of testing for test and comparator article defect trial sites was randomized for each animal prior to initiation of testing.

The test or comparator article was dispensed onto the defect (bleeding site) by pouring the powder directly from a pre-weighed vial. Initial tamponade was applied for approximately 30 seconds using digital pressure on a non-adherent wound dressing (i.e., Telfa ${ }^{\mathrm{TM}}$ Non-Adherent Dressing) placed over the powder. For negative control defect trial sites, the same process was followed but no powder was applied. Following the initial 30 seconds of tamponade, the dressing was removed. If hemostasis was not achieved after 30 seconds, tamponade was reapplied and maintained for an additional 30 seconds followed by another observation period. This process was repeated until effective hemostasis was achieved and the TTH was recorded, or until the testing period reached 10 minutes from the time of application. Each site was observed for free flow (active) bleeding for a minimum of 1 minute after the last period of tamponade that achieved a hemostatic end point.

TTH for each group was compared using both parametric (Student's $t$-test) and non-parametric (Mann-Whitney) tests. For sites where bleeding time surpassed the 10-minute time limit, the value of the time was treated as 10 minutes $(600 \mathrm{sec}-$ onds). Hemostatic success rate was compared using Fisher's exact test. All analyses were conducted with an alpha value of 0.05 being considered significant. In order to determine whether mass applied affected product efficacy, analysis of variance (ANOVA) was performed on TTH using treatment, mass applied, bleeding score, and animal as factors.

\section{Results}

\section{Liver punch biopsy trials}

Of the 70 defects/bleeding sites evaluated, $38.6 \%$ were classified as mild and $61.4 \%$ were classified as moderate bleeding; there were no defect sites classified as severe (pulsatile) bleeding. There was no significant difference in the distribution of level of bleeding between the four legs (SP, PC, AR, negative control).

Comparisons of hemostatic efficacy achieved within $\leq 10$ minutes indicated that SP provided $33 \%$ more effective hemostasis than PC ( $p=0.047)$ and the same rates of effective 
hemostasis as AR (Table 1). The negative control defective sites achieved $0 \%$ effective hemostasis, confirming that the model was appropriate to determine the efficacy of treatment application.

Comparisons of TTH were performed between the two masses of SP, AR, and PC that were tested (i.e., masses of 75 and $125 \mathrm{mg}$ ). There was no significant difference in TTH between the two masses that were evaluated $(p>0.5$ in all cases). Therefore, for comparisons between treatments, the two dosage levels were pooled. SP demonstrated a significantly lower TTH than either PC or AR $(p<0.001$, Figure 1 , Table 2). SP provided $89 \%$ faster median TTH than AR, and 93\% faster median TTH than PC.

An ANOVA evaluation of TTH using treatment (SP, AR, PC), mass applied $(75,125 \mathrm{mg})$, bleeding score (mild, moderate), and animal $(\mathrm{n}=5)$ as factors, indicated that only treatment

Table I Punch biopsy model effective hemostasis (hemostasis within 10 minutes)

\begin{tabular}{lllll}
\hline Treatment & $\begin{array}{l}\mathbf{7 5} \mathbf{~ m g} \\
\text { application }\end{array}$ & $\begin{array}{l}\text { I25 } \mathbf{~ m g} \\
\text { application }\end{array}$ & $\begin{array}{l}\text { Combined } \\
\text { data }\end{array}$ & $\begin{array}{l}\text { p-value* } \\
\text { (vs SP) }\end{array}$ \\
\hline $\begin{array}{l}\text { Negative } \\
\text { control }\end{array}$ & - & - & $0 / 10(0 \%)$ & $<0.001$ \\
AR & $10 / 10$ & $10 / 10$ & $20 / 20(100 \%)$ & 1.000 \\
PC & $7 / 10$ & $8 / 10$ & $15 / 20(75 \%)$ & 0.047 \\
SP & $10 / 10$ & $10 / 10$ & $20 / 20(100 \%)$ & - \\
\hline
\end{tabular}

Note: *For combined (75 and $125 \mathrm{mg}$ ) data.

Abbreviations: AR, Arista ${ }^{\mathrm{TM}}$ AH Absorbable Hemostatic Particles; PC, PerClot ${ }^{\circledR}$ Polysaccharide Hemostatic System; SP, Surgicel ${ }^{\circledR}$ Powder - Absorbable Hemostatic Powder. was a significant factor $(p<0.001)$, and TTH was independent of dosing and level of bleeding within the ranges tested.

\section{Liver abrasion trials}

Of the 85 defects/bleeding sites evaluated in five animals, $95.3 \%$ were classified as mild bleeding and $4.7 \%$ were classified as moderate; there were no defect/bleeding sites recorded as having severe (pulsatile) bleeding. There was no significant difference in the distribution of level of bleeding between the six study legs (negative control, SP, AR, PC, TP, IP).

Comparisons of hemostatic efficacy achieved within $\leq 10$ minutes indicated that SP provided over two times more effective hemostasis than PC ( $p=0.002)$ and over three times more effective hemostasis than AR, IP, and TP ( $p<0.001$, Table 3$)$. The negative control defective sites did not achieve effective hemostasis ( $0 \%$ efficacy), confirming that the model was valid for determining the efficacy of treatment application.

SP, with a median TTH of 90 seconds, achieved effective hemostasis significantly faster than all the comparator

Table 2 Punch biopsy model median TTH in seconds

\begin{tabular}{|c|c|c|c|c|}
\hline Treatment & $75 \mathrm{mg}$ & $125 \mathrm{mg}$ & $\begin{array}{l}\text { Both } \\
\text { masses }\end{array}$ & $\begin{array}{l}\text { P-value } \\
\text { (vs SP) }\end{array}$ \\
\hline AR & $285 \mathrm{~s}$ & $288 \mathrm{~s}$ & $286 \mathrm{~s}$ & $<0.001$ \\
\hline PC & $438 \mathrm{~s}$ & $415 \mathrm{~s}$ & $423 \mathrm{~s}$ & $<0.001$ \\
\hline SP & $30 s$ & $30 s$ & $30 s$ & - \\
\hline
\end{tabular}

Note: *For combined (75 and $125 \mathrm{mg}$ ) data.

Abbreviations: AR, Arista ${ }^{\mathrm{TM}}$ AH Absorbable Hemostatic Particles; PC, PerClot ${ }^{\circledR}$ Polysaccharide Hemostatic System; SP, Surgicel ${ }^{\circledR}$ Powder - Absorbable Hemostatic Powder; TTH, time-to-hemostasis.

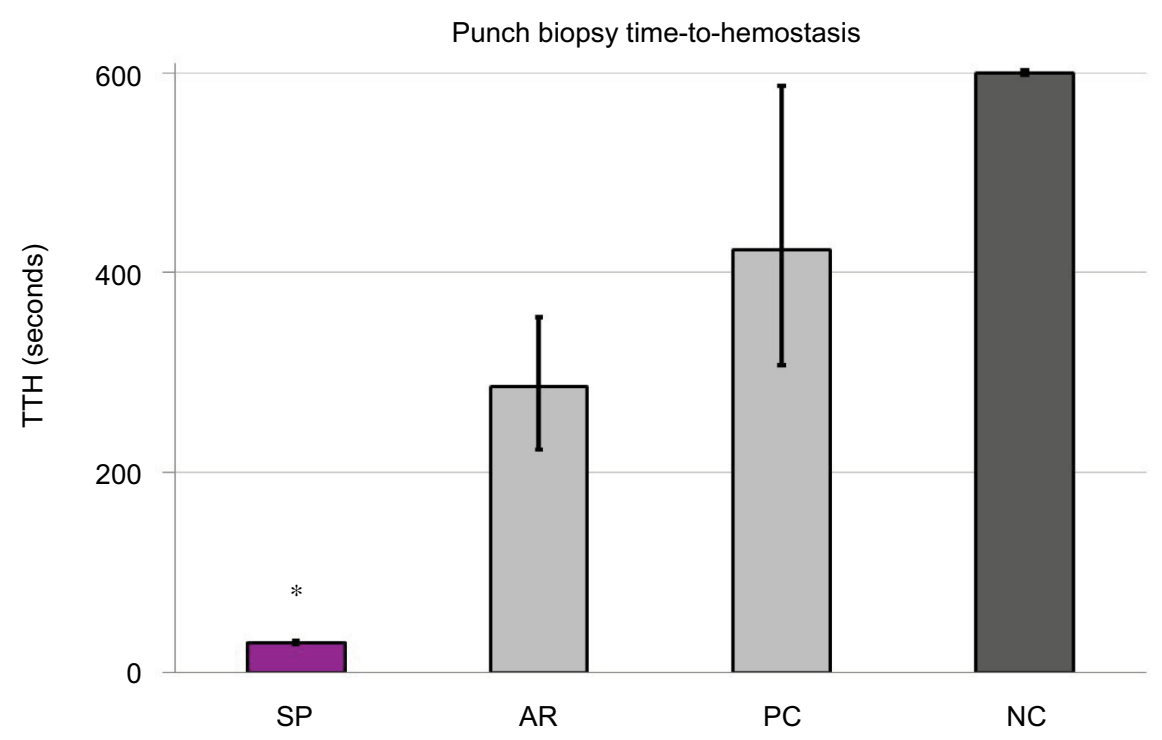

Figure I Punch biopsy median time-to-hemostasis for the test products and negative control. Error bars represent the $95 \%$ confidence interval of the median. *SP promoted hemostasis significantly faster than AR and PC $(p<0.001)$.

Abbreviations: SP, Surgicel ${ }^{\circledR}$ Powder - Absorbable Hemostatic Power; AR, Arista ${ }^{\text {TM }}$ AH Absorbable Hemostatic Particles; PC, PerClot ${ }^{\circledR}$ Polysaccharide Hemostatic System; NC, negative control (tamponade alone); TTH, time-to-hemostasis. 
products, which had median TTHs of $10 \mathrm{~min}(p<0.001$, Figure 2, Table 4). Since the longest time measured in this model was 10 minutes, SP achieved hemostasis at least $85 \%$ faster than the four comparator products.

\section{Discussion}

A recent review of the pattern of use of hemostatic agents suggests that these products have been used in over $30 \%$ of surgical procedures, and that their use is increasing. ${ }^{7}$ Presumably this trend reflects changing patient populations with comorbidities and those on chronic antiplatelet/anticoagulant therapies, increasing the need for this type of product. $^{7} \mathrm{~A}$ concomitant decrease in the rates of transfusion has been observed for many of the procedures using hemostatic agents.

Commonly used adjunctive topical hemostatic agents include oxidized/regenerated cellulose gelatin-based matrices

Table 3 Abrasion model effective hemostasis (hemostasis within 10 minutes)

\begin{tabular}{lll}
\hline Treatment & $\mathbf{3 0 0} \mathbf{~ m g}$ application & P-value (vs SP) \\
\hline Negative control & $0 / 10(0.0 \%)$ & $<0.001$ \\
AR & $4 / 15(27 \%)$ & $<0.001$ \\
IP & $4 / 15(27 \%)$ & $<0.001$ \\
PC & $7 / 15(47 \%)$ & 0.002 \\
TP & $3 / 15(20 \%)$ & $<0.001$ \\
SP & $15 / 15(100 \%)$ & - \\
\hline
\end{tabular}

Abbreviations: AR, Arista ${ }^{\mathrm{TM}}$ AH Absorbable Hemostatic Particles; IP, Instantaneous ${ }^{\circledR}$ Compound Microporous Polysaccharide Haemostatic Powder; PC, PerClot ${ }^{\circledR}$ Polysaccharide Hemostatic System; SP, Surgicel ${ }^{\circledR}$ Powder - Absorbable Hemostatic Powder; TP, Traumastem P Sterile Resorbable Haemostatic Powder. and polysaccharides. ${ }^{8}$ Cellulose is a linear polymer of D-glucose linked by $\beta$-glycosidic bonds. Regenerated cellulose is a more consistent form of cellulose made by dissolving the raw material and regenerating into a fiber. Chemical treatment can oxidize hydroxyl groups within the cellulose to carboxylic acid groups yielding ORC. The oxidation of cellulose increases the susceptibility to degradation by glycosidases and improves the hemostatic properties of the fibers. ${ }^{9}$ ORC is one of the most rapidly effective hemostatic agents used today. In a comparison performed in healthy humans, ORC produced faster coagulation than alginates, chitosan and collagen, and was also effective in subjects taking anticoagulants. ${ }^{10}$ Hemostatic costs have been shown to be substantially lower for ORC products, such as SF, when compared to other adjunctive hemostats. ${ }^{11,12}$

There are a few published comparisons among the base materials of the hemostatic agents used in this study, and

Table 4 Abrasion model median time-to-hemostasis in seconds

\begin{tabular}{lll}
\hline Treatment & $\mathbf{3 0 0} \mathbf{~ m g}$ & p-value (vs SP) \\
\hline AR & $600 \mathrm{~s}$ & $<0.001$ \\
IP & $600 \mathrm{~s}$ & $<0.001$ \\
PC & $600 \mathrm{~s}$ & $<0.001$ \\
TP & $600 \mathrm{~s}$ & $<0.001$ \\
SP & $90.0 \mathrm{~s}$ & - \\
\hline
\end{tabular}

Abbreviations: AR, Arista ${ }^{\mathrm{TM}}$ AH Absorbable Hemostatic Particles; IP, Instantaneous ${ }^{\circledR}$ Compound Microporous Polysaccharide Haemostatic Powder; PC, PerClot ${ }^{\circledR}$ Polysaccharide Hemostatic System; SP, Surgice ${ }^{\circledR}$ Powder - Absorbable Hemostatic Powder; TP, Traumastem P Sterile Resorbable Haemostatic Powder.

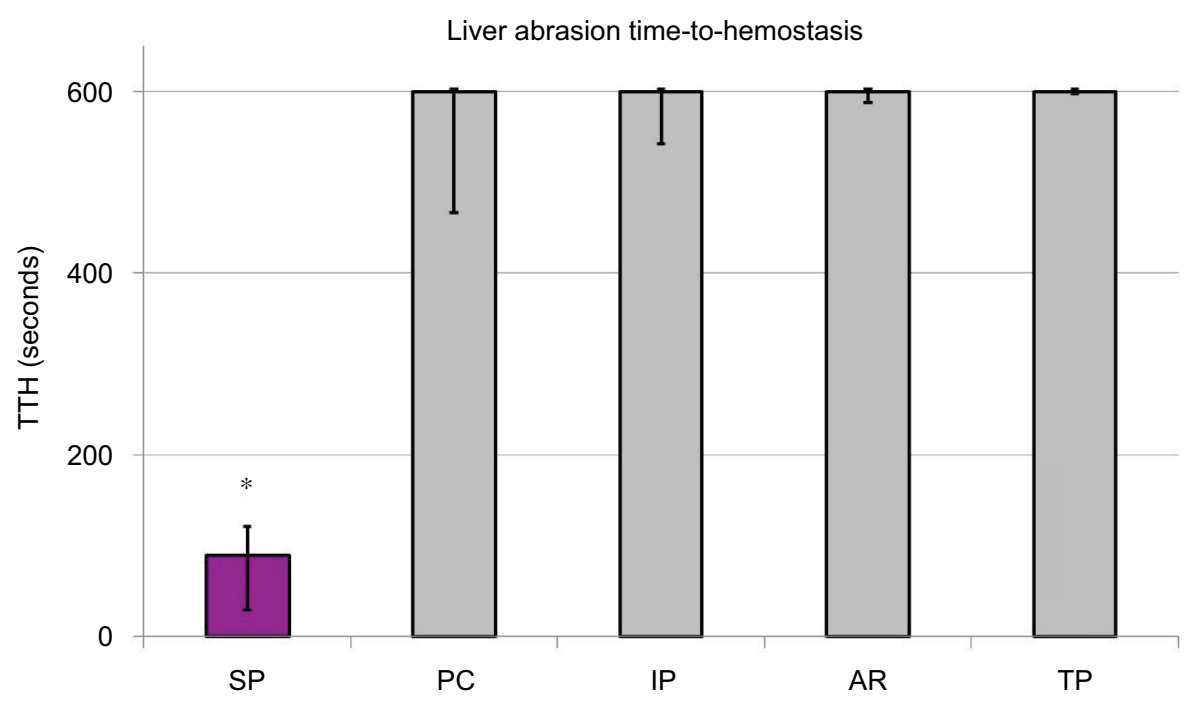

Figure 2 Liver abrasion median time-to-hemostasis for the test products and negative control. Times greater than 10 minutes were recorded as 10 minutes. Error bars represent the $95 \%$ confidence interval of the median. *SP promoted hemostasis significantly faster than PC, IP, AR and TP $(p<0.00 \mathrm{I})$.

Abbreviations: SP, Surgice ${ }^{\circledR}$ Powder - Absorbable Hemostatic Power; AR, Arista ${ }^{T M}$ AH Absorbable Hemostatic Particles; PC, PerClot ${ }^{\circledR}$ Polysaccharide Hemostatic System; IP, Instantaneous ${ }^{\circledR}$ Compound Microporous Polysaccharide Haemostatic Powder, TP, Traumastem P Sterile Resorbable Haemostatic Powder; TTH, time-to-hemostasis. 
none that include the powered form of SP. In a rat brain tissue defect model, SF (i.e., the fabric form) and AR appeared to perform equally well when compared to an untreated control with both products providing complete hemostasis within 1 minute, and there was no evidence of granuloma formation at implantation sites for either product when evaluated out to 28 days postoperatively. ${ }^{13}$ The fabric form of TP has been reported to be similar in bactericidal effectiveness and superior in terms of hemostasis to a fabric form of ORC, $\mathrm{SF},{ }^{14}$ but no comparisons have previously been published comparing powder forms of the two products. Apparently, the performance of PC and IP powders has until now not been compared to any of the Surgicel ${ }^{\circledR}$ family of absorbable hemostats.

All previously published evaluations of the Surgicel ${ }^{\circledR}$ hemostats used a fabric form of ORC; this is the first published in vivo study designed to evaluate a new powder form of SP and compare it to other commercially available powder hemostatic agents. The powder form promises to be useful in those applications where it would be impractical to position a fabric in place. As with the fabric form, SP is fully resorbable and may be left in place, although it is recommended to use only an amount sufficient to achieve hemostasis and remove any excess. In this study, commercially available hemostatic powders have been compared in two in vivo models of mild to moderate bleeding, liver punch biopsy and abrasion. While the punch biopsy model provides a higher intensity of initial bleeding, the abrasion model provides a more diffuse bleeding surface. However, both models are useful since both situations are commonly encountered in a clinical setting, and a hemostatic powder should be able to control mild bleeding in either case.

In the liver punch biopsy model, SP provided a significantly shorter TTH than the comparator products, $89 \%$ faster than AR and 93\% faster than PC. In fact, the median TTH for SP was 30 seconds, which is the minimum value that the model was designed to evaluate, while the actual TTH may have been lower. Effective hemostasis was defined as stopping bleeding within 10 minutes from the time of product application, and both SP and AR were $100 \%$ effective, while SP was significantly more effective than PC, which was only effectively hemostatic $75 \%$ of the time. Analysis of TTH versus mass applied showed no difference in hemostasis between treatment with 75 and $125 \mathrm{mg}$ for any of the products tested. Therefore, the amount of product used within this range is not expected to significantly affect the hemostatic efficacy and the amount of powder that provides adequate coverage can be determined by the user. A similar evaluation comparing bleeding intensity of mild and moderate bleeding demonstrated no difference in TTH outcomes based on the intensity of bleeding; therefore, it is anticipated that users may expect consistent hemostatic efficacy across a range of mild to moderate bleeding intensities.

In the liver abrasion model, SP also provided significantly shorter TTH values than the comparator products, $85 \%$ faster than AR, IP, PC, and TP. Since in this case the actual median time was greater than the maximum time measured (10 minutes) for all comparator products, the difference between SP and these comparator products is predicted to be even greater than $85 \%$. For effective hemostasis, SP was consistently $100 \%$ effective, and superior to the comparator products by margins of up to five times. No rebleeding was observed after the sites treated with SP were irrigated to remove the excess product that was not a part of the clot after hemostasis was achieved.

Severe bleeding was not studied in these models and the products were not evaluated for management of severe bleeding. SP and the other powdered hemostats evaluated in this study are indicated to assist in the control of capillary, venous, and small arterial hemorrhage when ligation or other conventional methods of control are impractical or ineffective ${ }^{15,16}$ or as a primary method of hemostasis for mild bleeding. ${ }^{17}$ For severe bleeding, other methods of hemostasis are recommended. As a result, these models can be considered to be relevant to the clinical conditions for which powdered topical hemostats are indicated.

In conclusion, in these two models of mild to moderate bleeding using adequate coverage of the bleeding sites, Surgice ${ }^{\circledR}$ Powder demonstrated faster and more effective hemostasis than comparator products, and this was independent of the amount of powder used.

\section{Disclosure}

All authors are employees of Ethicon, Inc., manufacturer of SP. The authors report no other conflicts of interest in this work.

\section{References}

1. Karkouti K, Dattilo KM. Perioperative hemostasis and thrombosis. Can J Anaesth. 2006;53(12):1260-1262.

2. Keshavarzi S, MacDougall M, Lulic D, Kasasbeh A, Levy M. Clinical experience with the surgicel family of absorbable hemostats (oxidized regenerated cellulose) in neurosurgical applications: a review. Wounds. 2013;25(6):160-167.

3. Hutchinson RW, George K, Johns D, Craven L, Zhang G, Shnoda P. Hemostatic efficacy and tissue reaction of oxidized regenerated cellulose hemostats. Cellulose. 2013;20(1):537-545.

4. Bruckner BA, Blau LN, Rodriguez L, et al. Microporous polysaccharide hemosphere absorbable hemostat use in cardiothoracic surgical procedures. J Cardiothorac Surg. 2014;9(1):134. 
5. Wang Y, Xu M, Dong H, et al. Effects of PerClot ${ }^{\circledR}$ on the healing of fullthickness skin wounds in rats. Acta Histochem. 2012;114(4):311-317.

6. Hutchinson RW, Werrlein S, Johns DB, Zhang G, Clymer JW, Kocharian $\mathrm{R}$. An in vivo comparison of hemostatic gelatin matrix products in a porcine spleen biopsy-punch model. Surg Technol Int. 2015;27:53-57.

7. Wright JD, Ananth CV, Lewin SN, et al. Patterns of use of hemostatic agents in patients undergoing major surgery. J Surg Res. 2014;186(1):458-466.

8. Gabay M, Boucher BA. An essential primer for understanding the role of topical hemostats, surgical sealants, and adhesives for maintaining hemostasis. Pharmacotherapy. 2013;33(9):935-955.

9. Pierce AM, Wiebkin OW, Wilson DF. Surgicel $®$ : its fate following implantation. J Oral Pathol. 1984;13(6):661-670.

10. Rembe JD, Böhm JK, Fromm-Dornieden C, et al. Comparison of hemostatic dressings for superficial wounds using a new spectrophotometric coagulation assay. J Transl Med. 2015;13(1):375.

11. Martyn D, Meckley LM, Miyasato G, et al. Variation in hospital resource use and cost among surgical procedures using topical absorbable hemostats. Clinicoecon Outcomes Res. 2015;7:567-574.
12. Martyn D, Kocharian R, Lim S, et al. Reduction in hospital costs and resource consumption associated with the use of advanced topical hemostats during inpatient procedures. J Med Econ. 2015:18(6): 474-481.

13. Ereth MH, Schaff M, Ericson EF, Wetjen NM, Nuttall GA, Oliver WC Jr. Comparative safety and efficacy of topical hemostatic agents in a rat neurosurgical model. Neurosurgery. 2008;63(4 Suppl 2): 369-372.

14. Lewis KM, Spazierer D, Urban MD, Lin L, Redl H, Goppelt A. Comparison of regenerated and non-regenerated oxidized cellulose hemostatic agents. Eur Surg. 2013;45(4):213-220.

15. Instructions for Use - SURGICEL Powder Absorbable Haemostatic Powder. Somerville, NJ: Ethicon, Inc; 2017.

16. Instructions for Use-ARISTA AH Absorbable Particles. Minneapolis, MN: Medafor Hemostatic Polymer Technologies; 2008.

17. Instructions for Use - PerClot Topical Hemostatic Particles. Kennesaw, GA: CryoLife Inc.; 2014.
Medical Devices: Evidence and Research

\section{Publish your work in this journal}

Medical Devices: Evidence and Research is an international, peerreviewed, open access journal that focuses on the evidence, technology, research, and expert opinion supporting the use and application of medical devices in the diagnosis, monitoring, treatment and management of clinical conditions and physiological processes. The identification of novel

\section{Dovepress}

devices and optimal use of existing devices which will lead to improved clinical outcomes and more effective patient management and safety is a key feature. The manuscript management system is completely online and includes a quick and fair peer-review system. Visit http://www. dovepress.com/testimonials.php to read real quotes from authors. 